\title{
On the generalization of the Stein-Weiss theorem for the ergodic Hilbert transform
}

\author{
by \\ LASHA EPHREMIDZE (Tbilisi)
}

\begin{abstract}
The Stein-Weiss theorem that the distribution function of the Hilbert transform of the characteristic function of $E$ depends only on the measure of $E$ is generalized to the ergodic Hilbert transform.
\end{abstract}

1. Introduction. Let $(X, \mathbb{S}, \mu)$ be a $\sigma$-finite measure space and let $\left(T_{t}\right)_{t \in \mathbb{R}}$ be an ergodic group of measure-preserving transformations on $X$. As usual the map $(x, t) \mapsto T_{t} x$ is assumed to be jointly measurable. If $f \in L(X)$, then the ergodic Hilbert transform of $f$ is defined by the formula

$$
\mathbb{H}(f)(x)=\lim _{\delta \rightarrow 0+} \frac{1}{\pi} \int_{\{\delta \leq|t| \leq 1 / \delta\}} \frac{f\left(T_{-t} x\right)}{t} d t .
$$

It is well known that the limit in (1) exists and consequently $\mathbb{H}(f)(x)$ is defined for a.a. $x \in X$ (see [4], [5]). If $T_{t}, t \in \mathbb{R}$, are the translations on the real line, $x \mapsto x+t$, and $f \in L(\mathbb{R})$, then we get the usual Hilbert transform

$$
H(f)(x)=\lim _{\delta \rightarrow 0+} \frac{1}{\pi} \int_{\{\delta \leq|t| \leq 1 / \delta\}} \frac{f(x-t)}{t} d t .
$$

We also consider the conjugate of $2 \pi$-periodic integrable (on $\mathbb{T}=(0,2 \pi)$ ) functions:

$$
\widetilde{f}(x)=\lim _{\delta \rightarrow 0+} \frac{1}{2 \pi} \int_{\{\delta \leq|t| \leq \pi\}} f(x-t) \cot \frac{t}{2} d t .
$$

As usual, in this case we may assume the function $f$ and its conjugate $\tilde{f}$ to be defined on the boundary of the unit circle.

The two theorems below which identify the distribution functions of the Hilbert transform and of the conjugate of $\mathbb{1}_{E}$, the characteristic function of a measurable set $E$, are well known and belong to Stein and Weiss [6].

2000 Mathematics Subject Classification: 28D10, 42A50.

Key words and phrases: ergodic Hilbert transform, Stein-Weiss theorem. 
Theorem A. Let $E \subset \mathbb{R}$ be a measurable set with finite Lebesgue measure, $m(E)<\infty$. Then

$$
\begin{aligned}
m\left\{x \in \mathbb{R}: H\left(\mathbb{1}_{E}\right)(x)>\lambda\right\} & =m\left\{x \in \mathbb{R}: H\left(\mathbb{1}_{E}\right)(x)<-\lambda\right\} \\
& =\Psi(m(E), \lambda), \quad \lambda \geq 0 .
\end{aligned}
$$

Theorem B. Let $E \subset \mathbb{T}$ be a measurable set. Then $m\left\{x \in \mathbb{T}: \tilde{\mathbb{1}}_{E}(x)>\lambda\right\}=m\left\{x \in \mathbb{T}: \widetilde{\mathbb{1}}_{E}(x)<-\lambda\right\}=\Phi(m(E), \lambda), \quad \lambda \geq 0$.

The original proof of the authors uses real methods, while there exist shorter proofs applying complex methods $([1],[3])$. The exact form of the functions $\Psi$ and $\Phi$ is also well known (see [6], [7]):

$$
\Psi(\xi, \lambda)=\frac{\xi}{\sinh (\pi \lambda)} \quad \text { and } \quad \Phi(\xi, \lambda)=2 \arctan \frac{\sin (\xi / 2)}{\sinh (\pi \lambda)} .
$$

In the present paper we try to generalize the Stein-Weiss theorem to the ergodic Hilbert transform. The similarity between the ergodic and the usual Hilbert transforms makes one believe that the generalization is possible (though we have not found it done elsewhere and the personal communications confirm that the result obtained is new). On the other hand there is no evident reason why an ergodic analog of such an exact quantitative theorem should remain true for every measure space.

The generalization we achieve is only for finite measure spaces, and the ergodic Hilbert transform behaves like the conjugate rather than the usual Hilbert transform in this case. It makes the constants simpler to assume below that the measure of the total space is $2 \pi$ instead of 1 .

THEOREM 1. Let $\left(T_{t}\right)_{t \in \mathbb{R}}$ be an ergodic flow of measure-preserving transformations on a finite measure space $(X, \mathbb{S}, \mu)$ with $\mu(X)=2 \pi$ and let $E \in \mathbb{S}$. Then

$$
\begin{aligned}
\mu\left\{x \in X: \mathbb{H}\left(\mathbb{1}_{E}\right)(x)>\lambda\right\} & =\Phi(\mu(E), \lambda) \\
& =\mu\left\{x \in X: \mathbb{H}\left(\mathbb{1}_{E}\right)(x)<-\lambda\right\}, \quad \lambda \geq 0 .
\end{aligned}
$$

Although we believe that the direct generalization of Theorem A should be true for the ergodic Hilbert transform on infinite measure spaces, the method of proof we propose for Theorem 1 fails to achieve this goal.

In the specific case where the $\left(T_{t}\right)_{t \in \mathbb{R}}$ is the group of rotations on the unit circle the ergodic Hilbert transform coincides with the conjugate, i.e. Statement 1 below holds. This means that the usual Hilbert transform of an integrable (on $\mathbb{T}$ ) $2 \pi$-periodic function (although the function is not integrable on $\mathbb{R}$ in this case, unless it is identically 0 , it can be proved by direct calculations that the limit in (2) exists for a.a. $x \in X$ anyway) is equal to its conjugate. This equation is usually proved by transforming the kernel $\frac{1}{t}$ into $\frac{1}{2} \cot \frac{t}{2}$ by means of a special way of summation (see [2]). The methods 
developed in the paper enable us to give a proof of this fact by direct calculations of integrals. This approach apparently has an independent interest and is presented in Section 4.

StATEMEnT 1. Let $(X, \mathbb{S}, \mu)=(\mathbb{T}, \mathcal{B}, m)$ be the unit circle and $\left(T_{t}\right)_{t \in \mathbb{R}}$ be the group of rotations on it, $T_{t}(x)=x+t(\bmod 2 \pi), x \in \mathbb{T}$. If $f \in L(\mathbb{T})$, then

$$
\mathbb{H}(f)(x)=\widetilde{f}(x)
$$

for a. a. $x \in \mathbb{T}$.

2. Notation and preliminary propositions. We say that $x \in X$ measures $A \in \mathbb{S}$ properly if

$$
\lim _{s \rightarrow \infty} \frac{1}{s} \int_{0}^{s} \mathbb{1}_{A}\left(T_{t} x\right) d t=\lim _{s \rightarrow \infty} \frac{1}{s} \int_{0}^{s} \mathbb{1}_{A}\left(T_{-t} x\right) d t=\frac{\mu(A)}{\mu(X)} .
$$

For any countable system $\left\{A_{n}\right\}_{n=1}^{\infty}$ of sets, it follows from the Ergodic Theorem that almost every $x \in X$ measures properly each $A_{n}$.

The principle value integral $Y=\lim _{\delta \rightarrow 0+} \int_{\{\delta<|t|<a\}} f(t) d t$ will be written as $Y=(\mathrm{P}) \int_{-a}^{a} f(t) d t$ and this will always mean that the right side of the equality exists and is equal to $Y$. If $f \in L(X)$, then

$$
\mathbb{H}^{d}(f)(x)=(\mathrm{P}) \int_{-d}^{d} \frac{f\left(T_{-t} x\right)}{t} d t
$$

and $\mathbb{H}(f)(x)=\lim _{d \rightarrow \infty} \mathbb{H}^{d}(f)(x)$ for a.a. $x \in X$.

The dilation and translation operators on $\mathcal{M}(\mathbb{R})$ (the measurable functions on $\mathbb{R}$ ) will be denoted by $D$ and $S$ respectively,

$$
D_{r} f(\xi)=f(r \xi), \quad S_{r} f(\xi)=f(\xi-r), \quad \xi, r \in \mathbb{R} .
$$

These operators satisfy certain commutation relations, $D_{r} S_{\varrho}=S_{\varrho / r} D_{r}$, and the translations commute with the Hilbert transform, $S_{r} H=H S_{r}$, and with the conjugate operator, $S_{r} \widetilde{f}=\widetilde{S_{r} f}$. Moreover, it easily follows from the change of variables in the integral that $D_{r} H(f)=H\left(D_{r} f\right), r>0$, and the boundary value property of the conjugate implies that if $n \in \mathbb{N}$, then $D_{n} \widetilde{f}=\widetilde{D_{n} f}$. Consequently, if $f \in L(\mathbb{T})$ and $x \in \mathbb{T}$ are such that $\widetilde{D_{n} f}(x / n)=D_{n} \widetilde{f}(x / n)=\widetilde{f}(x)$ for each $n=1,2, \ldots$ (a.a. $x \in \mathbb{R}$ will be such), then

$$
\begin{aligned}
\tilde{f}(x) & =\frac{1}{2 \pi}(\mathrm{P}) \int_{-\pi}^{\pi} D_{n} f\left(\frac{x}{n}-t\right) \cot \frac{t}{2} d t \\
& =\frac{1}{2 \pi}(\mathrm{P}) \int_{-\pi}^{\pi} D_{n} S_{-x} f(-t) \cot \frac{t}{2} d t
\end{aligned}
$$


We say that a sequence $\left\{h_{n}\right\}_{n=1}^{\infty}$ of functions is regularly distributed on an interval $(a, b)$ (with constant $c$ ) if $h_{n}$ is uniformly bounded, $\sup _{n}\left\|h_{n}\right\|_{\infty}$ $<\infty$, and

$$
\lim _{n \rightarrow \infty} \int_{\alpha}^{\beta} h_{n} d m=c(\beta-\alpha)
$$

for each $(\alpha, \beta) \subset(a, b)$. Obviously, if $h_{n}(t)$ is regularly distributed on $(a, b)$, then $h_{n}(\xi-t)$ will be regularly distributed on $(\xi-b, \xi-a)$ (with the same constant) and if $h(t)$ is a bounded periodic function, then $h_{n}(t)=h(n t), n=$ $1,2, \ldots$, will be regularly distributed on $\mathbb{R}$ (with constant $p^{-1} \int_{0}^{p} h d m$, where $p$ is the period of $h$ ).

It easily follows from (5) that if $A \in \mathbb{S}$ and $x$ measures $A$ properly, then the sequence of functions $h_{n}(t)=\mathbb{1}_{A}\left(T_{n t} x\right), n=1,2, \ldots$, is regularly distributed on $\mathbb{R}$ with constant $\mu(A) / \mu(X)$ : indeed,

$$
\int_{\alpha}^{\beta} h_{n}(t) d t=\frac{1}{n}\left(\int_{0}^{n \beta} \mathbb{1}_{A}\left(T_{t} x\right) d t-\int_{0}^{n \alpha} \mathbb{1}_{A}\left(T_{t} x\right) d t\right) \rightarrow \frac{\mu(A)}{\mu(X)}(\beta-\alpha) .
$$

If $[a, b]$ is a finite interval, let $\mathcal{F}[a, b]$ be the set of step functions,

$$
\mathcal{F}[a, b]=\left\{g: g=\sum_{i=0}^{j-1} l_{i} \mathbb{1}_{\left[a_{i}, a_{i+1}\right)}, a=a_{0}<a_{1}<\ldots<a_{j}=b\right\}
$$

and let $\overline{\mathcal{F}}[a, b]$ be its closure in the $L_{\infty}$ norm.

The lemma below follows immediately from the given definitions.

Lemma 1. If $g \in \overline{\mathcal{F}}[a, b]$ and a sequence of functions $h_{n}$ is regularly distributed on $(a, b)$ with constant $c$, then

$$
\lim _{n \rightarrow \infty} \int_{a}^{b} g h_{n} d m=c \int_{a}^{b} g d m .
$$

The following lemma can be proved in the same way as in the case where the sequence $\left\{F_{n}^{0}\right\}_{n=1}^{\infty}$ consists of one function. Therefore we omit its proof.

Lemma 2. Let $\left\{F_{n}^{i}\right\}_{n=1}^{\infty}, i=0,1$, be sequences of measurable functions on a space $X$ with finite measure, $\mu(X)<\infty$, such that the distribution functions of $F_{n}^{0}$ are convergent to some function $\varphi, \lim _{n \rightarrow \infty} \mu\left\{F_{n}^{0}>\lambda\right\}=$ $\varphi(\lambda)$ for each $\lambda \in \mathbb{R}$, and $\lim _{n \rightarrow \infty}\left(F_{n}^{1}(x)-F_{n}^{0}(x)\right)=0$ for a.a. $x \in X$. Then

$$
\lim _{n \rightarrow \infty} \mu\left\{F_{n}^{1}>\lambda_{0}\right\}=\varphi\left(\lambda_{0}\right)
$$

for every point $\lambda_{0}$ of continuity of $\varphi$.

3. Auxiliary lemmas. Let

$$
K_{1}(t) \equiv \frac{1}{\pi t} \quad \text { and } \quad K_{2}(t) \equiv \frac{1}{2 \pi} \cot \frac{t}{2}, \quad 0<|t| \leq \pi,
$$


so that $\int_{\{\delta<|t|<\pi\}} K_{i}(t) d t=0, i=1,2$, and

$$
\lim _{\delta \rightarrow 0+} \sup _{|t|<\delta}\left|K_{1}(t)-K_{2}(t)\right|=0 .
$$

LEMMA 3. Let functions $h_{n}, n=1,2, \ldots$, be regularly distributed on $(-\pi, 3 \pi)$ and let $\xi \in \mathbb{T}$. If

$$
F_{n}^{i}(\xi)=(\mathrm{P}) \int_{-\pi}^{\pi} h_{n}(\xi-t) K_{i}(t) d t, \quad i=1,2
$$

then

$$
\lim _{n \rightarrow \infty}\left|F_{n}^{1}(\xi)-F_{n}^{2}(\xi)\right|=0 .
$$

Proof. For each $\delta>0$, we have

$$
\begin{aligned}
F_{n}^{i}(\xi)= & (\mathrm{P}) \int_{-\delta}^{\delta} h_{n}(\xi-t) K_{i}(t) d t \\
& +\int_{\{\delta<|t|<\pi\}} h_{n}(\xi-t) K_{i}(t) d t, \quad i=1,2 .
\end{aligned}
$$

Since $\mathbb{1}_{\{\delta<|t|<\pi\}} K_{i}(t) \in \overline{\mathcal{F}}[-\pi, \pi]$ and $\int_{\{\delta<|t|<\pi\}} K_{i}(t) d t=0$, the second summand in (9) converges to 0 as $n \rightarrow \infty$ by Lemma 1. Thus, for each $\varepsilon>0$, if $\delta>0$ is small such that $\sup _{|t|<\delta}\left|K_{1}(t)-K_{2}(t)\right|<\varepsilon$ (see (7)), then

$$
\begin{aligned}
\limsup _{n \rightarrow \infty}\left|F_{n}^{1}(\xi)-F_{n}^{2}(\xi)\right| & =\limsup _{n \rightarrow \infty}\left|(\mathrm{P}) \int_{-\delta}^{\delta} h_{n}(\xi-t)\left(K_{1}(t)-K_{2}(t)\right) d t\right| \\
& \leq 2 \sup _{n}\left\|h_{n}\right\|_{\infty} \delta \varepsilon
\end{aligned}
$$

and (8) is proved since $h_{n}$ is uniformly bounded and $\varepsilon$ is arbitrary.

LEMMA 4. Let $\left\{h_{n}^{0}\right\}_{n=1}^{\infty}$ be regularly distributed on $(-\pi, 3 \pi)$ and suppose $h_{n}^{1}, n=1,2, \ldots$, are defined by

$$
h_{n}^{1}(t)= \begin{cases}h_{n}^{0}(t+2 \pi) & \text { when }-\pi<t<0 \\ h_{n}^{0}(t) & \text { when } 0 \leq t<2 \pi \\ h_{n}^{0}(t-2 \pi) & \text { when } 2 \pi \leq t<3 \pi\end{cases}
$$

If $\xi \in \mathbb{T}$ and

$$
F_{n}^{i}(\xi)=(\mathrm{P}) \int_{-\pi}^{\pi} h_{n}^{i}(\xi-t) K_{1}(t) d t, \quad i=0,1
$$

then

$$
\lim _{n \rightarrow \infty}\left|F_{n}^{1}(\xi)-F_{n}^{0}(\xi)\right|=0
$$


Proof. Take $\delta_{\xi}>0$ small such that $\left(\xi-\delta_{\xi}, \xi+\delta_{\xi}\right) \subset \mathbb{T}$. We have

$$
\begin{aligned}
F_{n}^{i}(\xi)= & (\mathrm{P}) \int_{-\delta_{\xi}}^{\delta_{\xi}} h_{n}^{i}(\xi-t) K_{1}(t) d t \\
& +\int_{\left\{\delta_{\xi}<|t|<\pi\right\}} h_{n}^{i}(\xi-t) K_{1}(t) d t, \quad i=0,1 .
\end{aligned}
$$

Clearly, $\left\{h_{n}^{1}\right\}_{n=1}^{\infty}$ will also be regularly distributed on $(-\pi, 3 \pi)$. Therefore, the second summand in (11) converges to 0 as $n \rightarrow \infty$ by Lemma 1 , and the first summands coincide when $i=0$ and 1 . Consequently, (10) is valid.

Remark. Note that in Lemmas 3 and 4 the $\operatorname{limits}_{n \rightarrow \infty} \lim _{n}^{i}(\xi), i=$ $0,1,2$, may not exist at all.

Corollary 1. If $h_{n}^{i}, F_{n}^{i}, n=1,2, \ldots, i=0,1$, are as in Lemma 4 and $F_{n}^{2}(\xi)=\widetilde{h_{n}^{1}}(\xi), \xi \in \mathbb{T}\left(h_{n}^{1}\right.$ is assumed to be $2 \pi$-periodic on $\left.\mathbb{R}\right)$, then

$$
\lim _{n \rightarrow \infty}\left|F_{n}^{2}(\xi)-F_{n}^{0}(\xi)\right|=0 .
$$

Proof. By Lemmas 4 and 3, we have $\lim _{n \rightarrow \infty}\left|F_{n}^{1}(\xi)-F_{n}^{0}(\xi)\right|=0$ and $\lim _{n \rightarrow \infty}\left|F_{n}^{2}(\xi)-F_{n}^{1}(\xi)\right|=0$ respectively. Hence, (12) holds.

Lemma 5. Let $(X, \mathbb{S}, \mu)$ be a finite measure space, $f, f_{n} \in \mathcal{M}(X), n=$ $1,2, \ldots$, and

$$
\lim _{n \rightarrow \infty} f_{n}=f \quad \text { a.e. }
$$

If $\lambda_{0}$ is a continuity point of the distribution function of $f, \lambda \mapsto \mu\{f>\lambda\}$, then

$$
\lim _{n \rightarrow \infty} \frac{1}{\gamma n} \int_{0}^{\gamma n} \mathbb{1}_{\left\{f_{n}>\lambda_{0}\right\}}\left(T_{t} x\right) d t=\frac{\mu\left\{f>\lambda_{0}\right\}}{\mu(X)}
$$

for a.a. $x \in X$, where $\gamma$ is any positive constant.

Proof. Let us show that

$$
\liminf _{n \rightarrow \infty} \frac{1}{\gamma n} \int_{0}^{\gamma n} \mathbb{1}_{\left\{f_{n}>\lambda_{0}\right\}}\left(T_{t} x\right) d t \geq \frac{\mu\left\{f>\lambda_{0}+\varepsilon\right\}}{\mu(X)}
$$

and

$$
\limsup _{n \rightarrow \infty} \frac{1}{\gamma n} \int_{0}^{\gamma n} \mathbb{1}_{\left\{f_{n}>\lambda_{0}\right\}}\left(T_{t} x\right) d t \leq \frac{\mu\left\{f>\lambda_{0}-\varepsilon\right\}}{\mu(X)}
$$

for a.a. $x \in X$, where $\varepsilon$ is any positive number. Since $\lambda_{0}$ is a continuity point of the distribution function of $f$, this will finish the proof of the lemma.

Put $A_{k}=\left\{x \in X: f(x)>\lambda_{0}+\varepsilon,\left|f_{n}(x)-f(x)\right|<\varepsilon\right.$ when $\left.n>k\right\}$. Evidently,

$$
\mu\left(A_{k}\right) \nearrow \mu\left\{f>\lambda_{0}+\varepsilon\right\} .
$$


At the same time $A_{k} \subset\left\{f_{n}>\lambda_{0}\right\}$ when $n>k$. Therefore, if $x$ is such that it measures properly all the sets $A_{k}, k=1,2, \ldots$, then

$$
\liminf _{n \rightarrow \infty} \frac{1}{\gamma n} \int_{0}^{\gamma n} \mathbb{1}_{\left\{f_{n}>\lambda_{0}\right\}}\left(T_{t} x\right) d t \geq \lim _{n \rightarrow \infty} \frac{1}{\gamma n} \int_{0}^{\gamma n} \mathbb{1}_{A_{k}}\left(T_{t} x\right) d t=\frac{\mu\left(A_{k}\right)}{\mu(X)}
$$

for each $k \geq 1$. Consequently, it follows from (15) that (13) is valid.

Inequality (14) is equivalent to

$$
\liminf _{n \rightarrow \infty} \frac{1}{\gamma n} \int_{0}^{\gamma n} \mathbb{1}_{\left\{f_{n} \leq \lambda_{0}\right\}}\left(T_{t} x\right) d t \geq \frac{\mu\left\{f \leq \lambda_{0}-\varepsilon\right\}}{\mu(X)}
$$

and if $A_{k}=\left\{x \in X: f(x) \leq \lambda_{0}-\varepsilon,\left|f_{n}(x)-f(x)\right|<\varepsilon\right.$ when $\left.n>k\right\}$, then $\mu\left(A_{k}\right) \nearrow \mu\left\{f \leq \lambda_{0}-\varepsilon\right\}$ and $A_{k} \subset\left\{f_{n} \leq \lambda_{0}\right\}$ when $n>k$. This enables us to prove (16) exactly in the same way as (13).

4. Proof of the main result. We start with the proof of Statement 1.

If $\left\|f_{n}-f\right\|_{L(X)} \rightarrow 0$, then $\mathbb{H}\left(f_{n}\right) \rightarrow \mathbb{H}(f)$ in measure since the operator $\mathbb{H}$ is of weak type $(1,1)$ (see [4], [5]), and the same is true for the conjugate operator. Hence, it is sufficient to prove (4) for characteristic functions. Let us reformulate Statement 1 for the real line taking into account this remark.

Statement $1^{\prime}$. Let $E \subset \mathbb{R}$ be a $2 \pi$-periodic measurable set. Then, for a.a. $x \in \mathbb{R}$,

$$
\widetilde{\mathbb{1}}_{E}(x)=H\left(\mathbb{1}_{E}\right)(x) .
$$

Proof. Suppose $x$ is such that

$$
\widetilde{\mathbb{1}}_{E}(x)=(\mathrm{P}) \int_{-\pi}^{\pi} D_{n} S_{-x} \mathbb{1}_{E}(-t) K_{2}(t) d t, \quad n=1,2, \ldots,
$$

(see (6)) and

$$
H\left(\mathbb{1}_{E}\right)(x)=\frac{1}{\pi} \lim _{a \rightarrow \infty}(\mathrm{P}) \int_{-a}^{a} S_{-x} \mathbb{1}_{E}(-t) \frac{1}{t} d t
$$

(a.a. $x \in \mathbb{R}$ will be such). If we take $a_{n}=\pi n, n=1,2, \ldots$, in the latter integral, then a simple change of variables shows that

$$
H\left(\mathbb{1}_{E}\right)(x)=\lim _{n \rightarrow \infty}(\mathrm{P}) \int_{-\pi}^{\pi} D_{n} S_{-x} \mathbb{1}_{E}(-t) K_{1}(t) d t .
$$

If we let $h(t) \equiv \mathbb{1}_{E}(x+t)$ and $h_{n}(t)=h(n t), n=1,2, \ldots$, then $h_{n}(t)$ is regularly distributed on $\mathbb{R}$ (with constant $m(E) / 2 \pi$ ) and $D_{n} S_{-x} \mathbb{1}_{E}(-t)=$ $h_{n}(-t)$. Hence, it follows from Lemma $3(\xi=0$ in this situation) that (17) holds. 
Proof of Theorem 1. Obviously, it is enough to prove (3) for all $\lambda \geq 0$ except a countable number. Therefore, we can assume without loss of generality that $\lambda$ is a continuity point of the distribution function of $\mathbb{H}\left(\mathbb{1}_{E}\right)$.

Let

$$
f_{n}(x)=\frac{1}{\pi}(\mathrm{P}) \int_{-\pi n}^{\pi n} \mathbb{1}_{E}\left(T_{-t} x\right) \frac{1}{t} d t, \quad x \in X .
$$

Since $f_{n} \rightarrow \mathbb{H}\left(\mathbb{1}_{E}\right)$ a.e., it follows from Lemma 5 that

$$
\mu\left\{\mathbb{H}\left(\mathbb{1}_{E}\right)>\lambda\right\}=\lim _{n \rightarrow \infty} \frac{1}{n} \int_{0}^{2 \pi n} \mathbb{1}_{\left\{f_{n}>\lambda\right\}}\left(T_{t} x\right) d t
$$

for a.a. $x \in X$. Fix $x \in X$ which measures $E$ properly and for which (18) holds. Put

$$
\begin{aligned}
h(t)=\mathbb{1}_{E}\left(T_{t} x\right), & h_{n}(t)=D_{n} h(t) & =h(n t), \\
F_{n}^{0}(\xi)=(\mathrm{P}) \int_{-\pi}^{\pi} h_{n}(\xi-t) K_{1}(t) d t, & F_{n}^{2}(\xi) & =\widetilde{h_{n}^{1}}(\xi)=(\mathrm{P}) \int_{-\pi}^{\pi} h_{n}^{1}(\xi-t) K_{2}(t) d t,
\end{aligned}
$$

for $\xi \in \mathbb{T}$, where $h_{n}^{1}$ is the restriction of $h_{n}$ to $\mathbb{T}$ (assumed to be continued on $\mathbb{R}$ periodically), $n=1,2, \ldots$

Since $\left\{h_{n}\right\}_{n=1}^{\infty}$ is regularly distributed on $\mathbb{R}$ (with constant $\mu(E) / 2 \pi$, see Section 2), it follows from Corollary 1 that for a.a. $\xi \in \mathbb{T}$ we have

$$
\lim _{n \rightarrow \infty}\left|F_{n}^{2}(\xi)-F_{n}^{0}(\xi)\right|=0 .
$$

Since $h_{n}^{1}$ is a characteristic function of some $E_{n}^{1} \subset \mathbb{T}$, by Theorem B, we have $m\left\{\xi \in \mathbb{T}: F_{n}^{2}(\xi)>\lambda\right\}=\Phi\left(m\left(E_{n}^{1}\right), \lambda\right)$ and therefore

$$
\lim _{n \rightarrow \infty} m\left\{\xi \in \mathbb{T}: F_{n}^{2}(\xi)>\lambda\right\}=\Phi(\mu(E), \lambda), \quad \lambda \geq 0
$$

(indeed, $m\left(E_{n}^{1}\right)=\int_{0}^{2 \pi} \mathbb{1}_{E}\left(T_{n t} x\right) d t=n^{-1} \int_{0}^{2 \pi n} \mathbb{1}_{E}\left(T_{t} x\right) d t \rightarrow \mu(E)$ ). Thus, we can apply Lemma 2 to conclude that

$$
\lim _{n \rightarrow \infty} m\left\{\xi \in \mathbb{T}: F_{n}^{0}(\xi)>\lambda\right\}=\Phi(\mu(E), \lambda) .
$$

We also have

$$
\begin{aligned}
\frac{1}{n} \int_{0}^{2 \pi n} \mathbb{1}_{\left\{f_{n}>\lambda\right\}}\left(T_{t} x\right) d t & =\frac{1}{n} m\left\{0<\xi<2 \pi n: f_{n}\left(T_{\xi} x\right)>\lambda\right\} \\
& =m\left\{0<\xi<2 \pi: f_{n}\left(T_{n \xi} x\right)>\lambda\right\} \\
& =m\left\{\xi \in \mathbb{T}: \frac{1}{\pi}(\mathrm{P}) \int_{-\pi n}^{\pi n} h(n \xi-t) \frac{1}{t} d t>\lambda\right\} \\
& =m\left\{\xi \in \mathbb{T}:(\mathrm{P}) \int_{-\pi}^{\pi} h_{n}(\xi-t) K_{1}(t) d t>\lambda\right\} \\
& =m\left\{\xi \in \mathbb{T}: F_{n}^{0}(\xi)>\lambda\right\} .
\end{aligned}
$$


Thus, it follows from (18) that

$$
\mu\left\{\mathbb{H}\left(\mathbb{1}_{E}\right)>\lambda\right\}=\lim _{n \rightarrow \infty} m\left\{\xi \in \mathbb{T}: F_{n}^{0}(\xi)>\lambda\right\}
$$

and, taking into account (19), the first equality in (3) is proved.

The second equality can be proved in an exactly analogous way.

\section{References}

[1] A. P. Calderón, Singular integrals, Bull. Amer. Math. Soc. 72 (1966), 427-465.

[2] A. P. Calderón and A. Zygmund, Singular integrals and periodic functions, Studia Math. 14 (1954), 249-271.

[3] J. B. Garnett, Bounded Analytic Functions, Academic Press, New York, 1981.

[4] K. E. Petersen, Ergodic Theory, Cambridge Univ. Press, Cambridge, 1983.

[5] -, Another proof of the existence of the ergodic Hilbert transform, Proc. Amer. Math. Soc. 88 (1983), 39-43.

[6] E. M. Stein and G. Weiss, An extension of a theorem of Marcinkiewicz and some of its applications, J. Math. Mech. 8 (1958), 263-284.

[7] O. D. Tsereteli, Metric properties of conjugate functions, Itogi Nauki i Tekhniki 7 (1975), 18-57 (in Russian).

A. Razmadze Mathematical Institute

1, M. Aleksidze Str.

380093 Tbilisi, Georgia

E-mail: lasha@rmi.acnet.ge

Received March 11, 2002

Revised version July 5, 2002

(4903) 